\title{
Chrysotoxine attenuates sevoflurane-induced neurotoxicity in vitro via regulating PI3K/AKT/GSK pathway
}

\author{
Jin Qiu ${ }^{1}$, Yi Zhang ${ }^{2}$, Mian Xie ${ }^{1, *}$
}

\author{
${ }^{1}$ Department of Anesthesiology, \\ Chongqing Hospital of TCM, 400021 \\ Chongqing City, Chongqing, China \\ ${ }^{2}$ Department of Anesthesiology, The \\ Second Affiliated Hospital of Zunyi \\ Medical University, 563000 Zunyi City, \\ Guizhou Province, China
}

\section{*Correspondence}

mianx7644@163.com

(Mian Xie)

\begin{abstract}
Objective: The aim of this study is to investigate the neuroprotective effect of chrysotoxine (CTX) on sevoflurane-treated nerve cells and uncover the potential regulation mechanism.

Methods: Nerve cells treated with sevoflurane and CTX were subjected to MTT and apoptotic detection. Cell apoptosis and oxidative stress were detected by flow cytometry (FCM) and ELISA assays. In addition, immunoblot assay was performed to study the signaling pathway affected by CTX treatment.

Results: CTX treatment promoted the cell viability and suppressed the apoptosis of sevoflurane-treated SH-SY5Y cells. In addition, CTX inhibited the sevoflurane-induced oxidative stress response and inflammatory response in nerve cells. Mechanically, CTX ameliorated neurotoxicity through activating the PI3K/AKT/GSK signaling pathway. Conclusion: Therefore, CTX can serve as a promising drug target for treating anesthetics-induced neurotoxicity.
\end{abstract}

\section{Keywords}

Sevoflurane; Chrysotoxine (CTX); Neurotoxicity; Apoptosis; PI3K/AKT/GSK pathway

\section{Introduction}

Inhaled anesthetics are used as sedatives for surgery and also cause neurotoxic reactions $[1,2]$. The neurotoxic reactions are usually manifested by postoperative cognitive decline and neurodegenerative diseases [3, 4]. Currently, sevoflurane is one of the most commonly used inhaled anesthetics. It has been well favored for its rapid onset, but it was proved to be able to induce an increase in neurotoxic effects [5]. Notably, Sevoflurane increased the expression of pro-inflammatory cytokine such as interleukin-6 (IL-6) and activated the nuclear factor- $\kappa \mathrm{B}$ (NF- $\kappa \mathrm{B})$ pathway [6]. In addition, the expression of oxidative stress factors was increased in isoflurane-treated human glioma cells [7].

Anesthesia induces the activation of pro-apoptotic proteins in the rat hippocampus, which may lead to mitochondrial membrane rupture, further leading to apoptosis [8-10]. In addition, anesthetics may induce oxidative stress and inflammation in the brain, resulting in neurotoxicity $[2,3,11,12]$. Therefore, suppressing the apoptosis of brain neurons is an important means to counteract neurotoxicity induced by anesthetics.

Chrysotoxine (CTX), a monomer isolated from Dendrobium officinalis, is a kind of biphenyl compounds [13]. It has been shown to be an effective free radical scavenger, which has neuroprotective effects [14]. CTX has a protective effect on SH-SY5Y cells suffering from neurotoxicity induced by MPP+. CTX can also protect SH-SY5Y cells from 6-OHDA toxicity through protecting mitochondrial and regulating NF$\kappa \mathrm{B}$ signaling pathway [15]. In addition, CTX also has a variety of activities such as mitochondrial protection, ROS inhibition, cell survival promotion and cell death suppression $[15,16]$.

In this study, we found CTX treatment promoted the cell viability and suppressed the cell apoptosis in sevoflurane-treated SH-SY5Y cells. In addition, CTX inhibited sevoflurane-induced oxidative stress response and inflammatory response in nerve cells. Mechanically, we believe that CTX ameliorated neurotoxicity through activating the PI3K/AKT/GSK pathway. Therefore, CTX can serve as a promising therapeutic target for treating sevoflurane-induced neurotoxicity.

\section{Materials and methods}

\subsection{Cell culture and treatment}

The SH-SY5Y neuroblastoma cells were obtained from ATCC and maintained in DMEM culture medium supplemented with $10 \%$ fetal bovine serum (FBS) and incubated at $37^{\circ} \mathrm{C}$ in a $5 \%$ $\mathrm{CO}_{2}$ incubator. For sevoflurane exposure, cells were kept in $4.1 \%$ sevoflurane using calibrated vaporizers (Datex-Ohmeda Inc., Madison, WI, USA) with flow of $95 \%$ air and $5 \% \mathrm{CO}_{2}$ by an anesthesia machine (Excel 210SE; Ohmeda Inc., Madison, WI, USA) for $2 \mathrm{~h}$, which was administrated for 3 consecutive days as described previously [17]. The concentrations of CTX 
given were $25,50,100 \mu \mathrm{M}$ during the process of cell treatment.

\subsection{MTT assay}

Cells were plated into the 96-well plates with a density of 800 cells/well and subsequently maintained for 48 hours upon the indicated treatment. Cells were then treated with MTT for 4 hours. Subsequently, the medium was discarded and the remaining cells were fully dissolved with $150 \mu \mathrm{L}$ DMSO. The OD value was measured at $490 \mathrm{~nm}$ wave length.

\subsection{Immunoblot}

The cells were homogenized with RIPA lysis buffer and about 30 ug proteins were separated by SDS-PAGE. After transferred onto PVDF membrane, membranes were sealed with $5 \%$ skim milk and subsequently conjugated with the following primary antibodies against $\beta$-actin (1 : 10000 dilution, A1978, Sigma), BNDF (1 : 1000 dilution, ab108319, Abcam), Bax (1 : 1000 dilution, ab32503, Abcam), Bcl-2 (1: 1000 dilution, ab182858, Abcam), cleaved-caspase 3 (1 : 1000 dilution; ab32042, Abcam), PI3K (1 : 1000 dilution, ab32089, Abcam), p-AKT ( $1: 500$ dilution, ab38449, Abcam), AKT (1: 1000 dilution, ab8805, Abcam), GSK3 $\beta$ (1 : 1000 dilution; ab32391, Abcam) and p-GSK3 $\beta$ (1: 1000 dilution; ab75814, Abcam) at $4{ }^{\circ} \mathrm{C}$ overnight. Then the membranes were subjected to HRP-conjugated secondary antibodies for $1 \mathrm{~h}$. Signals were visualized by an ECL kit.

\subsection{Caspase-3 activity detection}

The activity of the Caspase- 3 in the SH-SY5Y cells was detected with the caspase-3 activity detection kit (ab252897, Abcam) according to manufacturer's instructions.

\subsection{Assessment of antioxidant activity}

The levels of MDA, SOD, GSH were assessed by the detection kits of Nanjing Jiancheng Bio-engineering Institute (Jiangsu, China) in accordance to the manufacturer's instructions.

\subsection{Lactate dehydrogenase (LDH) release activity}

The experiment was based on previous research [4]. LDH release activity was detected with an LDH cytotoxicity detection kit (Clontech laboratories Inc., CA, USA), following the manufacturer's protocol. Briefly, $100 \mu \mathrm{L}$ of culture supernatant from each well were added with $100 \mu \mathrm{L}$ of the $\mathrm{LDH}$ reaction solution, and then incubated at room temperature for $30 \mathrm{~min}$. Then, the optical densities of the samples at $490 \mathrm{~nm}$ was measured using a microplate reader.

\subsection{Quantitative PCR}

The experiment was based on previous research [8]. Total RNAs were extracted by Trizol (Invitrogen, USA) reagent according to manufacturer's protocol. The reverse transcription was conducted with reverse transcriptase (M1701, Promega, Wisconsin, USA) to establish cDNA profiles of each sample. Quantitative PCR was conducted by using SYBR Ex Taq kit (Takara, Japan), and the expression levels of target genes were normalized to $\beta$ actin level. Primers used in this assay are listed below: $\beta$-actin: forward: $5^{\prime}$-ACGGCCAGGTCATCACTATTG$3^{\prime}$; reverse: 5'-TGGATGCCACAGGATTCCA-3'; TNF$\alpha$ forward: 5'-GAACTGGCAGAAGAGGCACT-3', reverse 5'-GGTCTGGGCCATAGAACTGA-3'; IL-6 forward 5'-CTGATGCTGGTGACAACCAC-3', IL-6 reverse: $\quad 5^{\prime}$-CAGAATTGCCATTGCACAAC-3'; IL-1b: forward $5^{\prime}$-GCATCAACTTTGTGGGAATG- ${ }^{\prime}$, reverse: $5^{\prime}$ CGGTTCAAGCTTGCCAAA- ${ }^{\prime}$.

\subsection{Statistics}

GraphPad 7.0 was used for all the statistical analysis in this study. Data were expressed as mean \pm SD. Statistical analysis was performed by one-way ANOVA analysis, in which Bonferroni post hoc test was used. $p<0.05$ is considered as significant difference.

\section{Results}

\subsection{CTX treatment promoted the cell viability of sevoflurane-treated nerve cells}

To explore the role of CTX in sevoflurane-induced neurotoxicity in nerve cells, MTT assay was performed to detect cell viability following sevoflurane exposure. As shown in Fig. 1A, sevoflurane exposure resulted in obvious defect in cell viability. CTX treatment rescued cell viability in a dose dependent manner (Fig. 1A). Besides, CTX treatment reversed the decreased expression of BDNF induced by sevoflurane treatment (Fig. 1B). Taken together, these results indicated CTX relieved the sevoflurane-induced neurotoxicity.

\subsection{CTX treatment suppressed the sevoflurane-induced neuronal cell apoptosis}

Then, the effects of CTX on the apoptosis of SH-SY5Y cells treated with sevoflurane were detected by using FCM, ELISA and Immunoblot assays. FCM analysis results showed that sevoflurane treatment obviously elicited the nerve cell apoptosis, suggesting its neurotoxic effect. Importantly, the treatment of CTX $(25,50$, and $100 \mu \mathrm{M})$ suppressed the sevofluraneinduced apoptosis in a dose dependent manner (Fig. 2A). Caspase-3, a marker of cell apoptosis, can be used for detecting apoptosis. Compared with control group, caspase- 3 activity of cell in sevoflurane treatment group was significantly increased (Fig. 2B). CTX treatment dramatically counteracted the increasing effect of sevoflurane on caspase-3 activity of SHSY5Y cells (Fig. 2B). Immunoblot assays results revealed that sevoflurane treatment leaded to obvious high Bax and cleavedcaspase 3 expression and low Bcl-2 expression (Fig. 2C). As expected, CTX-treatment reversed the alteration of Bax, cleaved-caspase 3 and Bcl-2 expression induced by sevoflurane, suggesting that CTX treatment suppressed the sevoflurane induced cell apoptosis in nerve cells. 
A

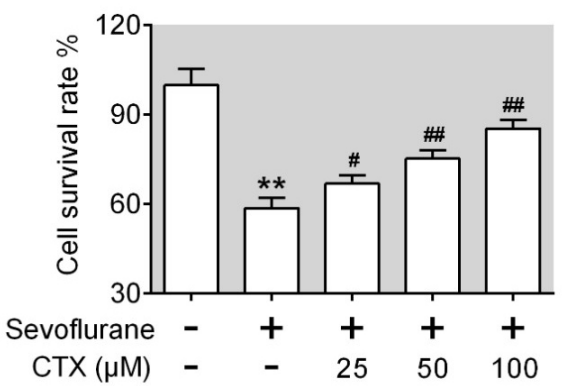

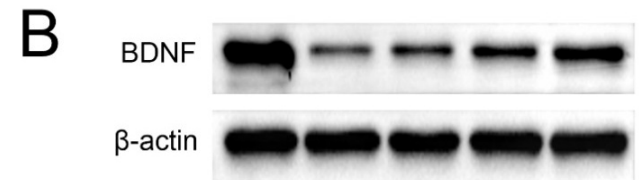

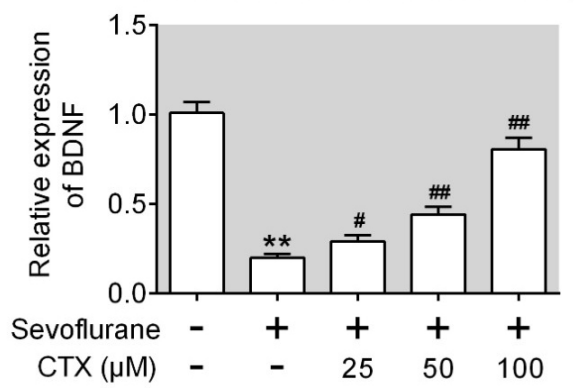

FIGURE 1. CTX treatment promoted the survival of sevoflurane-treated nerve cells. (A) The cell viability in the indicated groups. (B) The expression of BDNF in control, sevoflurane, sevoflurane + CTX (25 $\mu \mathrm{M})$, sevoflurane + CTX (50 $\mu \mathrm{M})$, sevoflurane + CTX $(100 \mu \mathrm{M})$ groups. Three independent experiments were performed. ${ }^{* *}, p<0.01$ vs control group, \#, $p$ $<0.05$, \#\#, $p<0.01$ vs sevoflurane group.

A

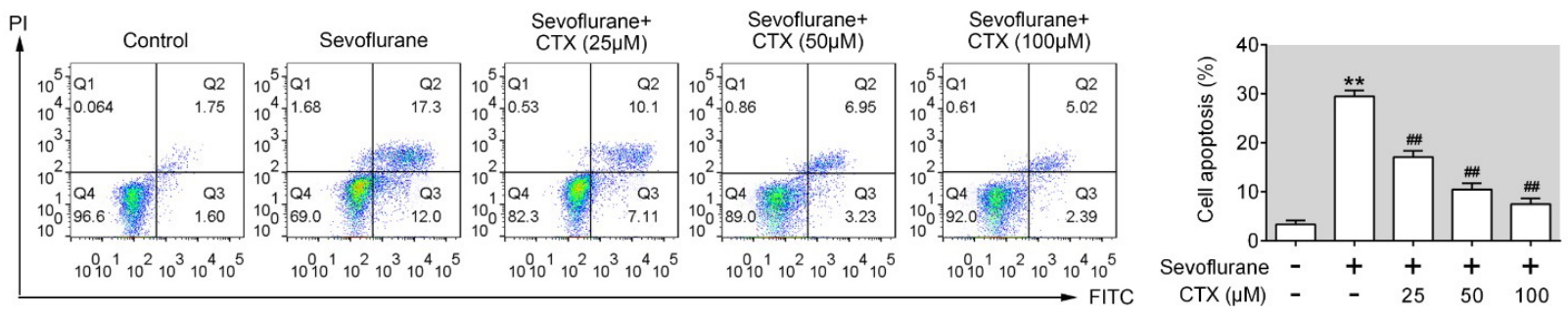

B

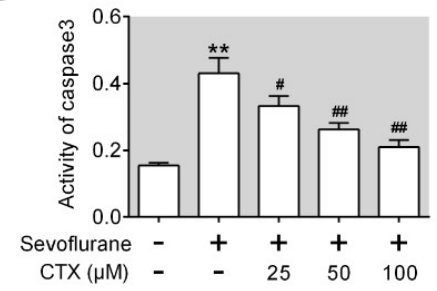

C

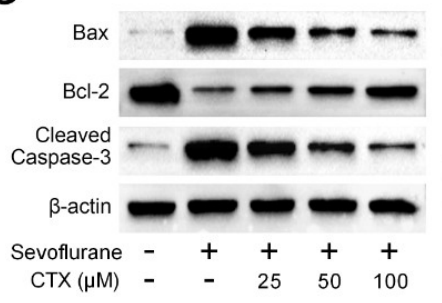

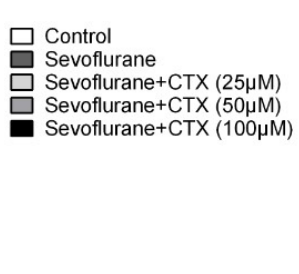

F I G U RE 2. CTX treatment suppressed the apoptosis of sevoflurane-induced SH-SY5Y cells. (A) Cell apoptotic ratio in the indicated groups. (B) The activity of caspase 3 in the indicated groups. (C) The level of Bcl-2, cleaved caspase 3 and Bax in control, sevoflurane, sevoflurane + CTX $(25 \mu \mathrm{M})$, sevoflurane + CTX $(50 \mu \mathrm{M})$, sevoflurane + CTX $(100 \mu \mathrm{M})$ groups. Three independent experiments were performed. ${ }^{* *}, p<0.01$ vs control group, $\#, p<0.05, \# \#, p<0.01$ vs sevoflurane group.

\subsection{CTX inhibited sevoflurane-induced oxidative stress response in nerve cells}

To examine the antioxidant effect of CTX, the level of SOD, GSH and MDA were detected in nerve cells treated with sevoflurane and CTX. Compared with Control group, expression of SOD and GSH in sevoflurane-treated group were significantly decreased, whereas MDA was markedly enhanced in the sevoflurane-treated group (Fig. 3A). CTX treatment totally reversed the oxidative stress response dramatically (Fig. 3A,B,C). Furthermore, sevoflurane leaded to a sharp increase in LDH release and CTX treatment alleviated the alterations (Fig. 3D). These data suggested CTX treatment effectively exerted an antioxidative effect on sevoflurane treated nerve cells.

\subsection{CTX inhibited the inflammatory response in nerve cells}

To detect the inflammation status in sevoflurane treated cells, the mRNA and protein level of TNF- $\alpha$, IL- 6 , and IL- $1 \beta$ were detected in nerve cells. TNF- $\alpha$, IL- 6 , and IL- $1 \beta$ level were elevated by sevoflurane treatment. Following CTX treatment, TNF- $\alpha$, IL-6, and IL-1 $\beta$ mRNA and protein level were significantly reduced in a dose dependent manner (Fig. 4A,B). These results suggest that CTX decreased sevoflurane-induced cytokine expression in nerve cells, indicating alleviating the inflammatory response. 
A

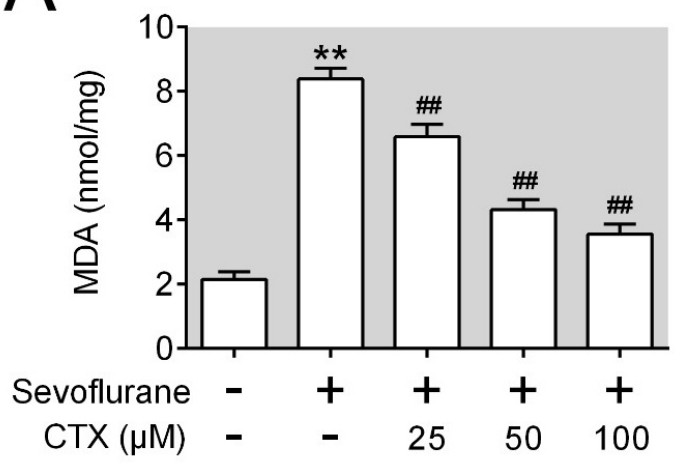

C

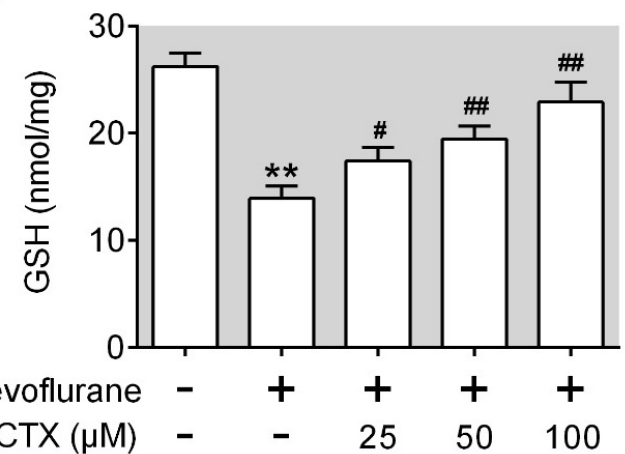

B

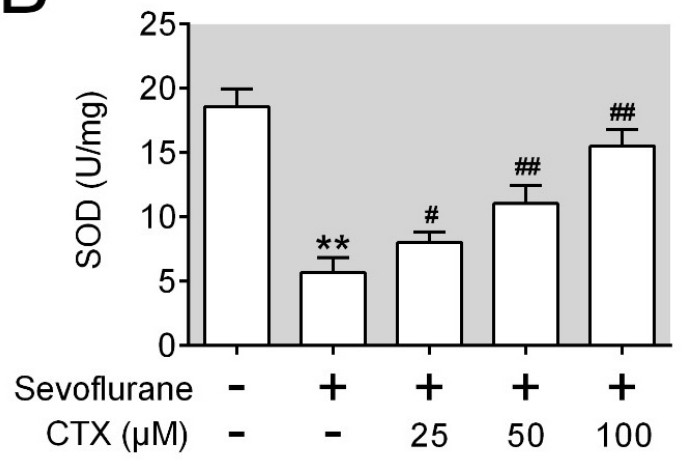

$\mathrm{D}$

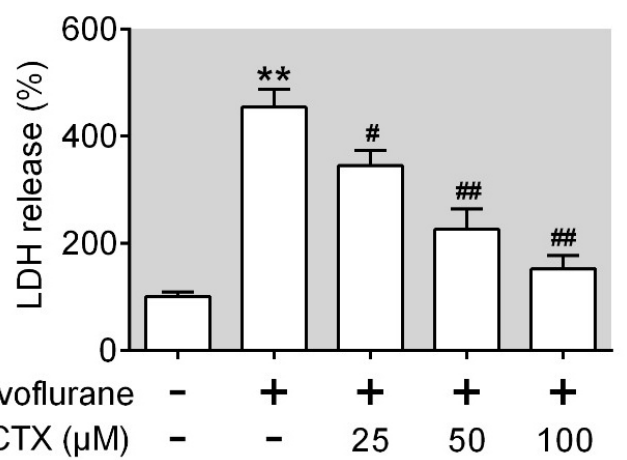

F I G U R E 3. CTX inhibited sevoflurane-induced oxidative stress response in nerve cells. (A, B, C, D) MDA, SOD, GSH, LDH level in the indicated groups were detected with respective ELISA kit. Three independent experiments were performed. **, $p<0.01$ vs control group, \#, $p<0.05, \# \#, p<0.01$ vs sevoflurane group.

\subsection{CTX ameliorated neurotoxicity through activation of the PI3K/AKT/GSK pathway}

PI3K/AKT pathway affected the pathogenesis of anestheticsinduced nerve injury. Thus, we speculated CTX might exert anti-apoptotic and anti-inflammatory effect through regulating PI3K/AKT/GSK signaling pathway. To prove the hypothesis, the expression level of PI3K, p-AKT, AKT, p-GSK-3 $\beta$ and GSK- $3 \beta$ was analyzed in nerve cells. In sevoflurane treated cells, PI3K, p-AKT and p-GSK-3 $\beta$ were obviously inhibited in nerve cells, which was significantly ameliorated by CTX treatment (Fig. 5). Thus, these findings suggested that CTX improved neurotoxicity through activating the PI3K/ AKT /GSK signaling pathway.

\section{Discussion}

In the last few years, it is generally believed that general anesthetics have a reversible effect on the central nervous system [18]. Anesthetics will not produce any toxic and side effects to central nervous system once they are completely metabolized, and the central nervous system can be restored to its initial state [19]. More studies have shown that general anesthetics have obvious neurotoxic effects on the brain tissues of developing animals [20]. In vivo and in vitro experiments have confirmed that exposure to isoflurane and sevoflurane, which are common clinical general anesthetics, can cause toxic changes in the nervous system [21]. Continued use of narcotic drugs can cause extensive neuronal death and certain neurological sequelae. Sevoflurane $(2.5 \%)$ treatment in neurodevelopmental primates can induce and maintain effective anesthesia and lead to changes in gene expression that results in neuronal damage [22]. In this study, we successfully constructed a sevoflurane-induced neuron injury model and explored an effective treatment to alleviate the neurotoxicity. We found that CTX, a traditional Chinese medicine extract, could effectively alleviate the neurotoxicity induced by sevoflurane.

The various biological activities of CTX have been widely revealed [16]. CTX has the anti-tumor activity in several types of tumors [13]. The inhibitory effect of CTX on cancer cell metastasis has been proved [23]. Previous studies have demonstrated CTX has a neuroprotective effects on neuro cells [24]. CTX suppressed 6-hydroxydopamine induced apoptosis in SH-SY5Y cells via mitochondria protection and the regulation of NF- $\kappa$ B pathway [15]. Additionally, CTX could decrease the neurotoxicity in dopaminergic SH-SY5Y cells [16]. Similarly, we found CTX attenuated sevofluraneinduced neurotoxicity in vitro in this study. We successfully constructed a cell model of sevoflurane-induced neurotoxicity. CTX improved the cell viability of nerve cells treated by 

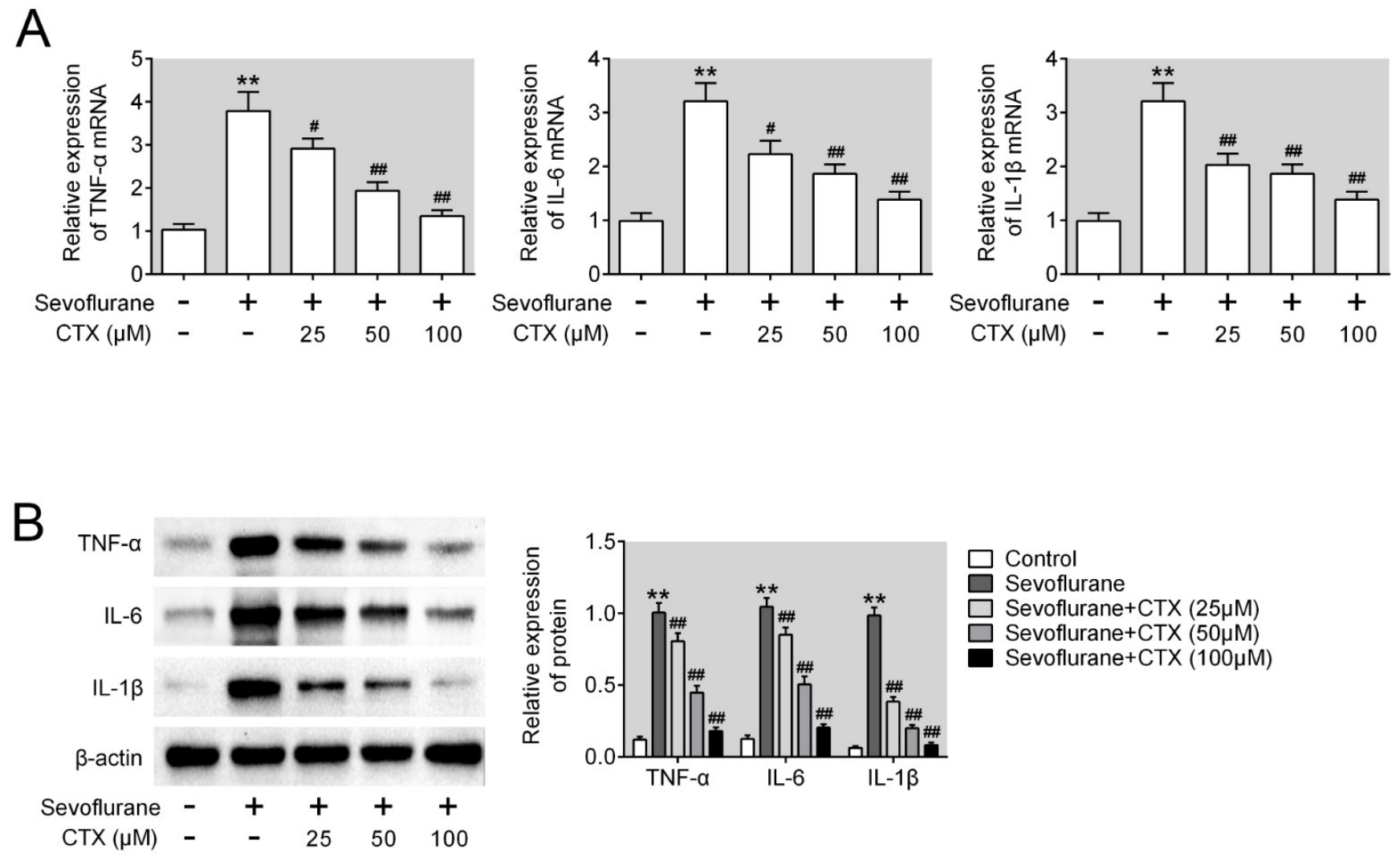

F I G U R E 4. CTX inhibited inflammatory response in nerve cells. (A, B) The mRNA and protein level of TNF-a, IL-6 and IL-1b in control, sevoflurane, sevoflurane + CTX $(25 \mu \mathrm{M})$, sevoflurane + CTX $(50 \mu \mathrm{M})$, sevoflurane + CTX $(100 \mu \mathrm{M})$ groups. Three independent experiments were performed. ${ }^{* *}, p<0.01$ vs control group, $\#, p<0.05$, \#\#, $p<0.01$ vs sevoflurane group.
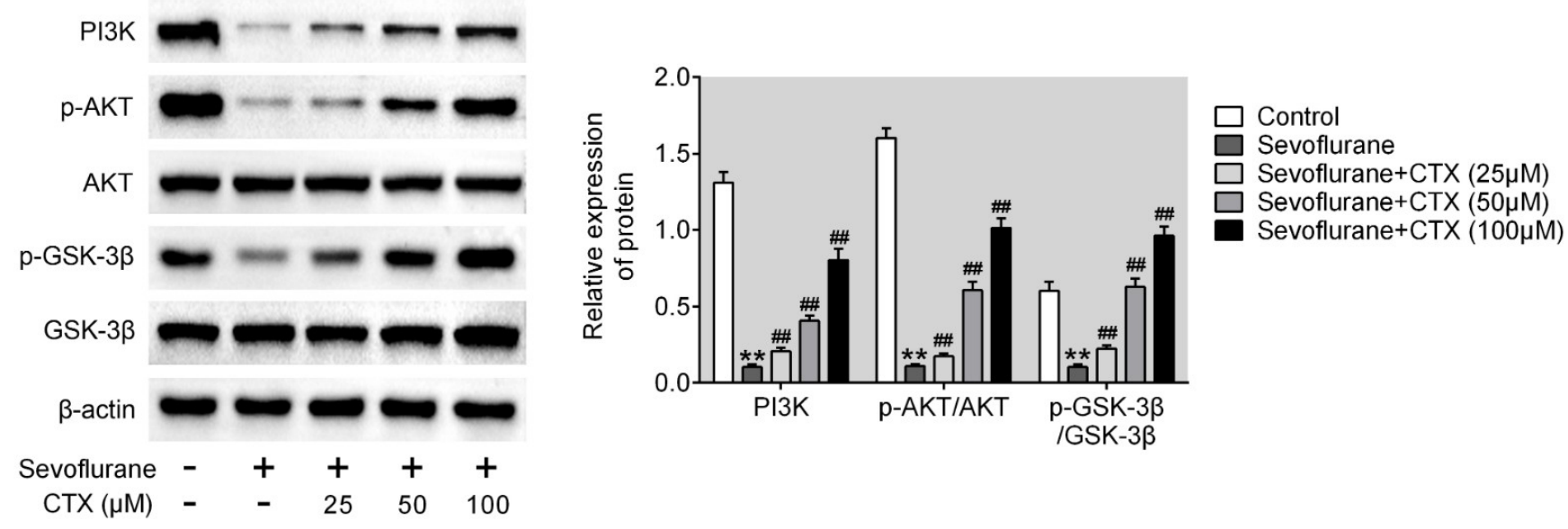

F I G URE 5. CTX ameliorated neurotoxicity through the activation of PI3K/AKT/GSK signaling pathway. The protein level of PI3K, AKT and GSK in control, sevoflurane, sevoflurane + CTX $(25 \mu \mathrm{M})$, sevoflurane + CTX $(50 \mu \mathrm{M})$, sevoflurane + CTX $(100 \mu \mathrm{M})$ group detected by immunoblot assay. Three independent experiments were performed. **, $p<0.01$ vs control group, \#\#, $p<0.01$ vs sevoflurane group.

sevoflurane. In addition, suggested that CTX counteracted the sevoflurane-induced cell apoptosis and ROS production revealed by FCM assays and ELISA assays. Moreover, CTX suppressed the inflammatory response induced by sevoflurane. These findings, together with the previous studies, confirmed the neuroprotective effects of CTX.

This study gave the evidence that CTX attenuated sevoflurane-induced neurotoxicity through the inhibition of apoptosis. In fact, several anesthetics could induce the activation of several pro-apoptotic proteins in hippocampus, further leading to apoptosis [25]. Consistent with previous study, anesthetics also induced oxidative stress and inflammation, resulting in the neurotoxicity in brain. Therefore, suppressing the apoptosis is critical to combat neurotoxicity induced by anesthetics. As expected, CTX attenuated sevoflurane-induced neurotoxicity via inhibiting 
apoptosis.

The immunoblot assays confirmed that CTX attenuated sevoflurane-induced neurotoxicity via regulating PI3K/AKT/GSK pathway [16]. It has been widely reported that the PI3K/AKT signaling pathway can affect the pathogenesis of anesthetics-induced nerve injury [13]. This pathway also regulates a variety of cellular processes, including inflammatory response, oxidative stress, and apoptosis, which is was further confirmed by our findings [26]. A variety of drugs can exert neuroprotective effects through this pathway, which also suggests that this pathway can be a potential target for the treatment of related diseases.

It is worth noting that free radicals have a wide range of effects on the nervous system [21]. In this study, we found that CTX attenuated sevoflurane-induced neurotoxicity, and the free radicals scavenging ability of CTX on in vivo should be further studied.

In conclusion, we successfully constructed a cell model of sevoflurane-induced neurotoxicity, and found CTX treatment promoted the cell survival and suppressed the sevofluraneinduced apoptosis in nerve cells. CTX could also inhibit sevoflurane-induced oxidative stress response and inflammatory response. Mechanically, CTX ameliorated neurotoxicity via targeting the PI3K/AKT/GSK signaling pathway. We therefore thought CTX could serve as a promising therapeutic target for treating sevoflurane-induced neurotoxicity.

\section{AUTHOR CONTRIBUTIONS}

JQ and YZ designed the study, supervised the data collection, MX analyzed the data, interpreted the data, MX, YZ and MX prepare the manuscript for publication and reviewed the draft of the manuscript. All authors have read and approved the manuscript.

\section{ETHICS APPROVAL AND CONSENT TO PARTICIPATE}

Not applicable.

\section{ACKNOWLEDGMENT}

Thanks to all the peer reviewers for their opinions and suggestions.

\section{FUNDING}

This research received no external funding.

\section{CONFLICT OF INTEREST}

The authors declare no conflict of interest.

\section{REFERENCES}

[1] Jildenstål P, Widarsson Norbeck D, Snygg J, Ricksten S, Lannemyr L. Cerebral autoregulation in infants during sevoflurane anesthesia for craniofacial surgery. Pediatric Anesthesia. 2021; 31: 563-569.
[2] Ward CG, Loepke AW. Anesthetics and sedatives: Toxic or protective for the developing brain? Pharmacological Research. 2012; 65: 271-274.

[3] Bittner EA, Yue Y, Xie Z. Brief review: anesthetic neurotoxicity in the elderly, cognitive dysfunction and Alzheimer's disease. Canadian Journal of Anaesthesia. 2011; 58: 216-223.

[4] León-Valenzuela Á, Román Malo C, González López M, Sánchez Palacios J, Sánchez Tarifa P, Del Pino Algarrada R. Sevoflurane sedation protocol in children with cerebral palsy undergoing botulinum toxin-A injections. Rehabilitacion. 2021; S0048-7120(20)30114-6. (In Spanish)

[5] Jiang W, Wang Q, Yajing Liao YL, Sun Y, Yang R. The effect of sevoflurane on the spatial recall ability and expression of apolipoprotein $\mathrm{E}$ and beta amyloid in the hippocampus in rats. Cellular and Molecular Biology. 2020; 66: 35-43.

[6] Zuo Y, Chang Y, Thirupathi A, Zhou C, Shi Z. Prenatal sevoflurane exposure: Effects of iron metabolic dysfunction on offspring cognition and potential mechanism. International Journal of Developmental Neuroscience. 2021; 81: 1-9.

[7] Kim JH, Oh AY, Choi YM, Ku SY, Kim YY, Lee NJ, et al. Isoflurane decreases death of human embryonic stem cell-derived, transcriptional marker Nkx2.5+ cardiac progenitor cells. Acta Anaesthesiologica Scandinavica. 2011; 55: 1124-1131.

[8] Şimşek HO, Kocatürk O, Demetoğlu U, Gürsoytrak B. Propofol based total intravenous anesthesia versus sevoflurane based inhalation anesthesia: the postoperative characteristics in oral and maxillofacial surgery. Journal of Cranio-Maxillofacial Surgery. 2020; 48: 880-884.

[9] Zhao S, Chen F, Wang D, Han W, Zhang Y, Yin Q. NLRP3 inflammasomes are involved in the progression of postoperative cognitive dysfunction: from mechanism to treatment. Neurosurgical Review. 2020.

[10] Wang Y, Yin CP, Tai YL, Zhao ZJ, Hou ZY, Wang QJ. Apoptosis inhibition is involved in improvement of sevoflurane-induced cognitive impairment following normobaric hyperoxia preconditioning in aged rats. Experimental and Therapeutic Medicine. 2021; 21: 203.

[11] Piao M, Wang Y, Liu N, Wang X, Chen R, Qin J, et al. Sevoflurane Exposure Induces Neuronal Cell Parthanatos Initiated by DNA Damage in the Developing Brain via an Increase of Intracellular Reactive Oxygen Species. Frontiers in Cellular Neuroscience. 2020; 14: 583782.

[12] Tang XL, Wang X, Fang G, Zhao YL, Yan J, Zhou Z, et al. Resveratrol ameliorates sevoflurane-induced cognitive impairment by activating the SIRT1/NF-kappaB pathway in neonatal mice. Journal of Nutritional Biochemistry. 2020; 90: 108579 .

[13] Bhummaphan N, Pongrakhananon V, Sritularak B, Chanvorachote P. Cancer Stem Cell-Suppressing Activity of Chrysotoxine, a Bibenzyl from Dendrobium pulchellum. The Journal of Pharmacology and Experimental Therapeutics. 2018; 364: 332-346.

[14] Fan J, Guan L, Kou Z, Feng F, Zhang Y, Liu W. Determination of chrysotoxine in rat plasma by liquid chromatography-tandem mass spectrometry and its application to a rat pharmacokinetic study. Journal of Chromatography. B, Analytical Technologies in the Biomedical and Life Sciences. 2014; 967: 57-62.

[15] Song JX, Shaw PC, Sze CW, Tong Y, Yao XS, Ng TB, et al. Chrysotoxine, a novel bibenzyl compound, inhibits 6-hydroxydopamine induced apoptosis in SH-SY5Y cells via mitochondria protection and NFkappaB modulation. Neurochemistry International. 2010; 57: 676-689.

[16] Song J, Shaw P, Wong N, Sze C, Yao X, Tang C, et al. Chrysotoxine, a novel bibenzyl compound selectively antagonizes MPP+, but not rotenone, neurotoxicity in dopaminergic SH-SY5Y cells. Neuroscience Letters. 2012; 521: 76-81.

[17] Xu L, Shen J, Dai S, Sun L, Chen X. Tetramethylpyrazine Attenuated Sevoflurane-Induced Neurotoxicity by Enhancing Autophagy through GPR50/CREB Pathway in SH-SY5Y Cells. The American Journal of Chinese Medicine. 2020; 48: 945-966.

[18] Nam DJ, Kim SH, Park SH, Lee H, Kang BB, Lee JH. Does desflurane need more irrigating-pump pressure for the visibility in arthroscopic shoulder surgery than sevoflurane? Anesthesia and Pain Medicine. 2020; 15: $35-40$.

[19] Zheng J, Du L, Zhang L. Seizure-like movements caused by residual sevoflurane inside the anesthesia machine: A case report. Medicine. 2021; 100: e24495.

[20] Orden C, Santos M, Ceprian M, Tendillo FJ. The effect of cannabidiol on sevoflurane minimum alveolar concentration reduction produced by 
morphine in rats. Veterinary Anaesthesia and Analgesia. 2021; 48: 74 81.

[21] Sung T, Lee D, Bang J, Choi J, Shin S, Kim T. Remifentanil-based propofol-supplemented vs. balanced sevoflurane-sufentanil anesthesia regimens on bispectral index recovery after cardiac surgery: a randomized controlled study. Anesthesia and Pain Medicine. 2020; 15: 424-433.

[22] Yamamoto H, Uchida Y, Chiba T, Kurimoto R, Matsushima T, Inotsume $\mathrm{M}$, et al. Transcriptome analysis of sevoflurane exposure effects at the different brain regions. PLoS ONE. 2020; 15: e0236771.

[23] Marra A, Rossi D, Pignataro L, Bigogno C, Canta A, Oggioni N, et al. Toward the identification of neuroprotective agents: g-scale synthesis, pharmacokinetic evaluation and CNS distribution of (R)-RC33, a promising Sigma1 receptor agonist. Future Medicinal Chemistry. 2016; 8: 287-295.

[24] Choi Y, Kim D, Kim Y, Yang S, Lee K, Ryu JH, et al. Synthesis and Evaluation of Neuroprotective Selenoflavanones. International Journal of
Molecular Sciences. 2015; 16: 29574-29582.

[25] Li H, Li J, Yu Q, Dai C, Gu J, Peng S, et al. Sevoflurane-induced neuronal apoptosis in neonatal mice is prevented with intranasal administration of insulin. American Journal of Translational Research. 2020; 12: 8175 8184 .

[26] Wainszelbaum MJ, Charron AJ, Kong C, Kirkpatrick DS, Srikanth P, Barbieri MA, et al. The Hominoid-specific Oncogene TBC1D3 Activates Ras and Modulates Epidermal Growth Factor Receptor Signaling and Trafficking. Journal of Biological Chemistry. 2008; 283: 13233-13242.

How to cite this article: Jin Qiu, Yi Zhang, Mian Xie. Chrysotoxine attenuates sevoflurane-induced neurotoxicity in vitro via regulating PI3K/AKT/GSK pathway. Signa Vitae. 2021;17(4):185-191. doi:10.22514/sv.2021.107. 